\title{
Coverillustration
}

\section{A well armed predator}

O nce considered a hazard to mariners, the mysterious and reclusive giant Pacific octopus (Enteroctopus dofleini) is a remarkable predator even if it doesn't attack boats. As one of the largest of the extant family of Octopodidae, this beautiful creature is an efficient and mostly nocturnal hunter that spends the day concealed in rocky caves in the northern Pacific. One specimen was weighed at $272 \mathrm{~kg}$ with a tip to tip spread of its arms of 9.6 metres, but most are in the $15 \mathrm{~kg}$ range with a much smaller arm spread. It is a primarily visual predator, relying upon sharp visual acuity honed with remarkable intelligence to subdue well fortified prey, and to protect itself against its own predators.

The octopus eye (an invertebrate eye) resembles a fish eye (a vertebrate eye) and is a good illustration of convergent evolution. On closer examination, however, there are substantial and critical differences illustrating that the eyes of octopuses and of fish do not have a common camera-style eye ancestor, but rather each evolved its individual eye separately.

Both the octopus and the fish eye have a camera-style eye with an iris, nearly circular lens, vitreous cavity, and photoreceptor cells lining the interior of the cavity. There the similarity ends. The octopus has no cornea and has a very different retinal anatomy when compared to that same fish. The retina is everted, meaning that the photoreceptive element is directly behind the lens with no interposed ganglion cells or other tissue to interfere with the image resolution. Hence, there is no blind spot and almost no other retinal cells within the eye. The equivalent neurological mechanisms are immediately behind the eye in an optic lobe containing amacrine and horizontal cells that resemble the vertebrate inner retina. The photopigment is receptive at approximately 475 $\mathrm{nm}$, making this remarkable creature colour blind. Futhermore, the photoreceptive pigment is in a rhabdomeric form similar to that found in insects. An octopus rhabdome consists of four rhabdomeres, each of which consists of precisely aligned and layered tubules that contain the photosensitive visual pigment. The rhabdomeres are paired, with one pair oriented horizontally and one pair oriented vertically so that each rhabdome has, in effect, two retinal units. Each pair of rhabdomeres (roughly

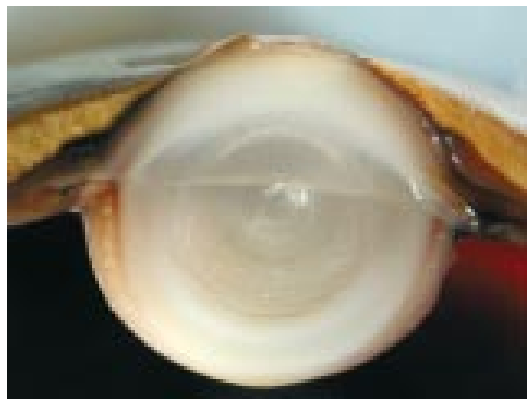

Note that there is no cornea; the interior of the eye communicates freely with sea water; and that the lens is formed in two halves.

a photoreceptor equivalent) subtends a visual angle of $1^{\circ} 3^{\prime}$ compared to our own $20-30^{\prime}$ in the fovea. The rhabdomes are separated from each other by pigment granules, and because of the orientation of the rhabdomeres, can easily perceive polarised light. The processes of the retinal receptors pass through a plexus, and then through a chiasm with several endings within an optic lobe in a complicated plexiform layer. This layer resembles our inner retina in that it has numerous amacrine cells, bipolar cells, and multiple communications within this lobe (Young JZ, Nature 1960; 186:836-9).

Not surprisingly, the lens is different from a fish lens too. The octopus lens has an opaque peripheral equatorial septum that divides the lens approximately in half (see Fig). This septum extends slightly into the lens and supplies structural support but minimally restricts light entry into the eye. Focus and accommodation (up to 14 dioptres) are achieved by an anterior-posterior movement of the spherical lens similar to the fish eye, in that the focal length is very short and the pupil can be large, thus creating a bright image in this compact eye. The horizontal pupil is unusual in a predator but apparently there is a good rationale in this species. When dilated the pupil is large and round. When the octopus is diurnally active the pupil will constrict to the slit seen on the cover This horizontal slit has been shown to limit the luminance without limiting the acuity as if pulling a blind in your house on a sunny day. The eyes are located on the sides of the head with a very limited, if any, binocular visual field. Most investigations of the octopus attack would suggest that these attacks are uniocularly guided. The visual system is coordinated by the connections through the ventral optic commissure, allowing the octopus to access memory units on the contralateral hemisphere as well as coordinate activity of all eight arms.

Hunting strategies are complex and include camouflage and ambush, stalking, chasing, and other more sophisticated techniques. But, mere capture of a well armoured crustacean, such as a crab, does not automatically kill it. The octopus accomplishes the kill by drilling a hole, with its tongue-like radula, in the crab's carapace, and injecting its venomous saliva. Although poorly understood, the poisonous saliva is used to dispatch the prey and begin the digestion, as well. Some octopus species are known to inject their enzymatic poison through the eye since it is the weakest point in the carapace and allows for the most rapid entry into the body.

These playful and highly intelligent invertebrates are a relatively recent product of cephalopod evolution, especially when compared to their ancient relative, the Nautilus (BJO cover, Dec 2000). Palaeontologists believe that the octopods first appeared about 65 million years ago perhaps just after the Cretaceous extinction. The decapods (squid) probably evolved first, followed by the octopods.

Curiosity and respect have swirled about the octopus like the concentrated ink it can discharge. Most octopods can change colour although not generally as magnificently as some of the decapods (see BJO cover, January 2001). It is the intelligence and learning capacity this animal exhibits that is perhaps the most fascinating element of its natural history. It has been known to catch and eat sharks in captivity, solve puzzles, open jars, and solve mazes (Roland Anderson, personal communication). In an incredible illustration of chicanery, one octopus has been videotaped climbing out of its container to traverse a laboratory floor to reach separately housed prey, consuming the prey, and returning to its original tank.

So with intelligence, good vision, camouflage, flexibility, and multiple powerful appendages, you can see this is truly a well armed predator.

\section{R Schwab irschwab@ucdavis.edu}

Photographs by the author.

Thanks to Roland Anderson, $\mathrm{PhD}$, at the Seattle Aquarium for assistance and counsel, and the opportunity to photograph, and be handled by, Billye, seen on the cover. 